That the sulphenic acid radical can decompose in this manner is indicated by the production, with the liberation of hydrogen sulphide, of glyoxylic acid from dithiodiglycollic acid, and of phenylglyoxylic acid from dithiodiphenylglyoxylic acid ${ }^{3}$. Keratin is also known to evolve hydrogen sulphide under the action of steam, and to lose 50 per cent of its sulphur when treated with sodium hydroxide ${ }^{4}$.

On the assumption that the cystine disulphide cross-linkages must be severed before hair can be removed rapidly from hides, the above hypothesis explains why fresh lime liquors are not efficient depilators, unless they contain reducing agents, for example, sodium sulphide. These substances reduce the sulphenic acid side-chains to cysteine side-chains and thus prevent the generation of aldehyde groups. The unexpected ability of aliphatic primary amines ${ }^{5}$ (especially methylamine) to facilitate depilation by fresh lime liquors (free from reducing agents) may also be due to the prevention of cross-linking, since these amines could condense with, and mask, the aldehyde groups arising by the sulphenic acid decomposition.

\section{H. Phillitps.}

\section{Wool Industries}

Research Association, Torridon,

Leeds, 6.

\footnotetext{
1 Marriott, J. Int. Soc. Leather Trades Chemists, 12, 216 (1928).

Speakman, NATURE, 182, 930 (1933).

3 Schöberl, Annalen, 507, 111 (1934); Ber., 67B, 1545 (1934).

- Harris, Bur. Stand. J. Res., 15, 63 (1935).

- McLaughlin, Highberger and Moore, J. Amer. Leather Chem. A8soc., 22, 345 (1927).
}

\section{Association and Dissociation Reactions of Thyro- globulin}

From their ultracentrifugal studies on thyroglobulin, Heidelberger and Pedersen ${ }^{1}$ found that solutions of this protein within its $p \mathrm{H}$ stability region ${ }^{2}$ are fairly homogeneous (Fig. 1). The sedimentation picture shows, in addition to a predominating component consisting of molecules having a molecular weight of about 700,000 , a small amount of heavier and lighter molecules with poorly defined sedimentation boundaries. These molecules were assumed to have been formed respectively by the dissociation and association of the molecules comprising the predominating component. It was noticed that on dilution the number of lower molecular weight molecules increased.

In the study of the stability of thyroglobulin being carried out by means of the ultracentrifuge, I have been able to confirm the previous work. I find, how. ever, that this protein dissociates in dilute solutions provided a number of other conditions are fulfilled. Furthermore, I find that the same factors are concerned in the association of thyroglobulin into molecules of higher molecular weight. Finally, I conclude that the number and size of molecules present in a given solution of thyroglobulin may be defined by the following set of variables : protein concentration, salt concentration, temperature, dielectric constant, $p \mathrm{H}$ and the time the solution has been standing under a given set of conditions.

Those conditions which favour the dissociation of thyroglobulin are as follows: low protein concentration, low salt concentration, elevated temperature, high dielectric constant (glycine and urea were added to the thyroglobulin solutions in this set of experiments), a $p \mathrm{H}$ displaced from the isoelectric point (only values on the alkaline side, but still within its $p H$ stability region, have been studied). These are the same conditions which favour the ionization of the protein.

On the other hand, those conditions which favour the association of thyroglobulin are the opposite or the same as those which minimize the ionization of the protein.

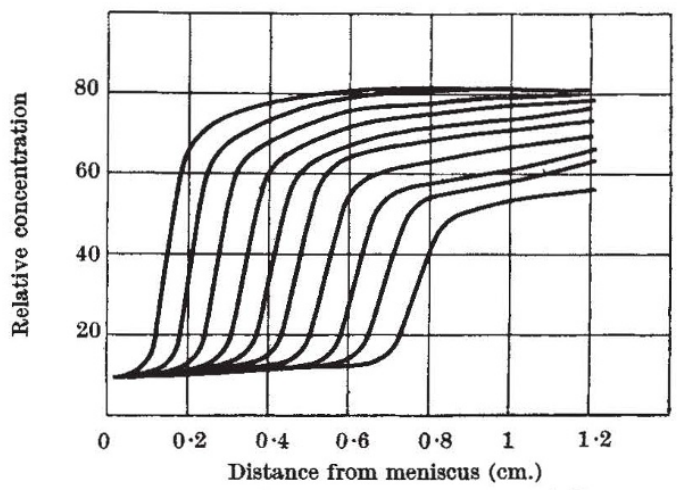

Fra. 1. Sedimentation diagram of thyroglobulin prepared essentially to reference 3 .

When placed under the proper combination of conditions, thyroglobulin dissociates with a slow and measurable rate into a series of components of lower molecular weight having well-defined sedimentation boundaries (Fig. 2). I have been able to reverse the reaction, resynthesising the original molecule from the dissociated fragments. Moreover, under the proper conditions, thyroglobulin will associate in due course into higher molecular weight components having definite sedimentation boundaries.

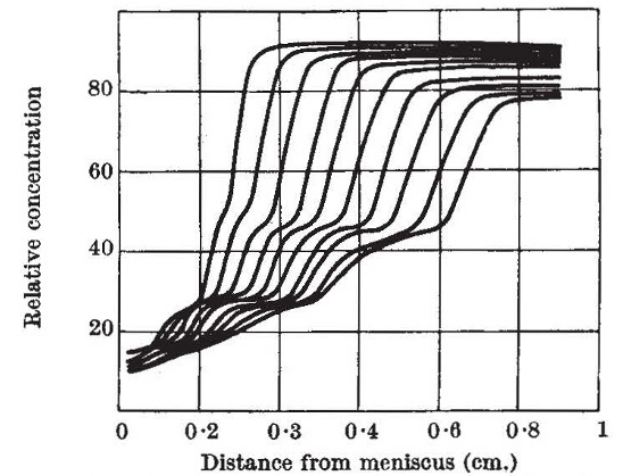

FIG. 2. Sedimentation diagram of dissociated thyroglobulin.

These factors alone cannot account for the mechanism of thyroglobulin economy within the body, for under the conditions existing in the living cell the rates of dissociation and association would be very slow.

\section{Harold P. Lundgren.}

Laboratory of Physical Chemistry, University, Uppsala. May 13.

${ }^{1}$ M. Heidelberger and K. O. Pedersen, J. Gen. Physiol., 19, 95 (1935).

M. Heidelberger and T. Svedberg, Science, 80, 414 (1934).

M. Heidelberger and W. Palmer, J. Biol. Chem., 101, 433 (1933). 